\begin{abstract}
This paper introduces Radix Intelligence, a new definition and integrative model of intelligence developed within the overarching system of Psychobionomy (Petrides, 2019). Following a discussion of misconceptions and pitfalls plaguing existing models of intelligence, with emphasis on the IQ literature, I point at Radix Intelligence as the primal energy underpinning mind activity in its entirety. All existence unfolds according to the laws of Radix Intelligence, whose nature is collective, impersonal, and non-local. Within the confines of the personalized human mind, the self-construct, a latent structure within the Thinking stage of the Psychobionomic system, refracts the unitary flow of Radix Intelligence into a manifold of major traits, including trait cognitive, trait emotional, and trait social intelligence, among others. In the context of elucidating Radix Intelligence, I offer a radical reinterpretation of the so-called "crud factor" and also touch on the issue of common method variance. Theoretical implications of Radix Intelligence are discussed with reference to the need for dynamic integration across the whole field of differential psychology, and practical implications with dual reference to the potential for establishing a new field of subconscious psychometrics and the pressing urgency for the individual to transition from a psychology of becoming to a psychology of being.
\end{abstract}

Keywords: Psychobionomy; intelligence; theoretical psychology; philosophy of mind; personality; crud factor; common method variance; subconscious psychometrics. 
RADIX INTELLIGENCE 2

\section{Radix Intelligence: A New Definition and Integrative Model of Intelligence}

\section{Introduction}

Prevailing psychometric conceptions of intelligence are woefully narrow and incomplete. They center on the view that each individual is in possession of some degree of a certain quality called "intelligence" that basically resides in the individual's brain and genes. Proponents of this view argue that this psychological quality, mystifyingly arising from physical matter, can be scientifically quantified through IQ tests. They further prescribe strict boundaries for this construct, despite the absence of consensually agreed attributes that can be consulted for setting such, with concomitant restrictions on psychometric instrumentation. The aim of this paper is to call attention to some misapprehensions and limitations of IQ-based conceptions of intelligence and, more importantly, to introduce a new conception that is truly general, complete, and invariant across time and place.

\subsection{Importance and limitations of IQ tests and the $g$ factor}

IQ is supposed to provide an age-adjusted index of intelligence and IQ tests play a cardinal role in psychometric conceptions of intelligence. Indeed, they are relied upon to define the very term. This means that the standard scientific practice of developing a theory to guide measurement is, in the case of IQ testing, turned on its head such that test properties and results need to be consulted in order to establish the meaning of the construct they are meant to be measuring. It is from this set of circumstances that Boring's (1923) infamous definition sprung: "intelligence is what intelligence tests measure". While catchy, this definition is fully circular and, therefore, of little scientific value. This leaves us with the enduring problem of how to define intelligence that has been plaguing the field for too long (Thorndike, 1921). 
Another important reason why IQ tests have a disproportionate influence on the conceptualization of intelligence is that they artificially restrict its content domain. The rationale here is that unless a variable can be shown to load onto the $g$ factor (Spearman, 1904), it cannot be admitted into the structure of cognitive abilities (Carroll, 1993). Whatever intelligence is, it can only include tasks that correlate with $g$ because the chief characteristic of IQ tests is that they are highly $g$ loaded (Jensen, 1998). This position, endorsed by many psychometricians, is not only circular (like Boring's definition), but at odds with reality. The $g$ factor is essentially a description of performance variation in mental ability tasks that has been stealthily elevated into a causal explanatory construct for which there is hardly any uncontroversial evidence after over 100 years of research (Howe, 1988). Extrapolating from performance on simplistic test tasks to an overarching explanatory construct constitutes terminally problematic reasoning for any position that simultaneously wishes to argue that intelligence has a genetic/physiological foundation (e.g., Jung \& Haier, 2007; Savage et al., 2018). To illustrate the challenge, just imagine if cardiologists were to attribute cardiac arrhythmia to a ... low standing on the construct of "heartbeat regulation ability"!

Efforts to discover possible mechanisms underlying IQ like, for instance, working memory, elementary cognitive processes, or brain and executive functions seem to be a long way from yielding any definitive answers (Goriounova \& Mansvelder, 2019). Despite decades of research, there is hardly any uncontested evidence that we have identified the fundamental constituent processes in IQ test performance and, furthermore, that these processes vary significantly between individuals in a manner consistent with their test performance. If anything, the evidence from the fields of cognitive psychology and neuroscience, to which IQ researchers have turned their attention for processes or brain regions that could substantiate the $\mathrm{g}$ factor, has 
actually supported a fractional view of cognition, split into multiple faculties, to the extent that any attempt at harmonizing IQ research with these two fields may require altogether sacrificing the notion of a general mental ability factor (Kovacs \& Conway, 2019).

There are other reasons why the $g$ factor, even if it were to be shown some day that it has real explanatory power, cannot constitute the principal dimension of individual differences that some might have envisaged it to be. Below, I present those that are most relevant for the purposes of this manuscript and refer readers to standard works on the subject for additional criticisms (e.g., Gould, 1996; Howe, 1997). The $g$ factor is purely a statistical concept, arising from patterns of covariation observed in large samples. It is not possible for something that has been derived from, and has no existence without, individual differences, to be intrapolated to the individual person; statistical concepts, like the $\mathrm{g}$ factor, reflecting relative rankings of individuals, may not be presumed to also retain a particularized existence (e.g., Fisher, Medaglia, \& Jeronimus, 2018; Lamiell, 2003).

Another reason impugning the psychological significance of $g$ is that it does not correlate strongly with fundamental human goals like happiness, probity, wisdom or non-ordinary success. Most of these variables are seen as either currently unmeasurable (e.g., wisdom) or as valued characteristics that are unrelated to $g$ because they are "non-cognitive". As regards the issue of measurement, just because a construct appears to be currently unmeasurable, that does not mean it can be casually set aside, pending the requisite psychometric advances. The related practice of partially measuring a construct that is only partially measurable at present and subsequently extrapolating the empirical findings to the construct as a whole or, worse still, amending the nature of the construct to align it with results obtained from an incomplete measurement process, must be rejected as thoroughly fallacious. 
Practices like the foregoing are ubiquitous in $g$ factor research, where any variable that does not load onto $g$ in a factor analysis is swiftly branded "non-cognitive". In light of the cardinal role of cognition in personality (e.g., Bandura, 1999; Kreitler, 2019; Revelle, 2012), this position is untenable, at least as far as personality is concerned. The line of argumentation that sees personality as "non-cognitive" appears to originate from the doctoral thesis of Charles Spearman's student, Edward Webb (1915), who set out to extend the remit of his mentor's $g$ factor into the germane area of character. Based on his specific measurements and the tacit need to safeguard the conceptual purity of the newly minted $g$ factor, Webb concluded it would be more appropriate for character traits (e.g., trustworthiness, conscientiousness, and perseverance) to be grouped under a second general factor, which he labelled " $w$ " for will or "persistence of motives". This factor was further clarified as "consistency of action resulting from deliberate volition or will" (Webb, 1915; p. 60) and subsequently became ingrained in the literature as a "non-cognitive" general factor.

Webb (1915) went on cautiously to argue that $w$ constitutes the second major dimension of individual differences and varies independently from $g$. This standpoint eventually became dominant, with intelligence and personality being absolutely distinguished and studied independently of each other (e.g., Hostee, 2001). However, while both Webb (1915) and Spearman (1927) tentatively suggested that $g$ and $w$ are empirically independent, they were united in conceptualizing them as distinct aspects of the same entity - mental energy. This is the same kind of awkward theorizing - admittedly introduced during the breakneck breakthroughs and advances of that early era - that we would later see in full force in the suggestion that the unitary construct of personality can somehow be reduced to five dimensions that are orthogonal between them. 
Psychometric tests purporting to measure intelligence while being unable to predict with practically useful precision the most desirable outcomes in life cannot, in my opinion, be taken as valid measures of any generally acceptable definition of intelligence. That is not to negate their predictive validity in relation to mundane criteria like formal scholastic achievement, getting and keeping a job, social conventionality, and perhaps living a little longer (e.g., Gottfredson, 1997). Millions of people excel in all these domains simultaneously, yet they are still narcissistic, depressed, and with a deep sense of lack of fulfillment. If IQ is to be vaguely interpreted as some composite index of learning and adaptation to the environment (Sternberg, 2000), then this raises the question of whether it is truly intelligent to learn how to adapt to a society that is suffering from a mental health crisis, coupled with diminishing faith in the prevailing paradigms for treating it (e.g., Joober, 2016).

A spate of evidence that high IQ is a significant risk factor for serious psychological maladaptation is yet another reason why a high standing on the $g$ factor cannot be seen as some unmitigated privilege in life. For instance, Karpinski, Kolb, Tetreault, and Borowski (2018) analyzed data from a large sample of American Mensans and found that high IQ was associated with affective, Autism Spectrum, and autoimmune disorders, among others. In a large metaanalysis of 96 studies covering over 80,000 participants, Cassidy, Yang, Kapczinski, and Passos (2017) found that higher IQ was a significant risk factor for committing suicide. Lopez, Stahl, and Tchanturia (2010) showed that people suffering from anorexia nervosa have significantly higher IQs than average, while Penney, Miedema, and Mazmanian (2015) linked high IQ to cognitive processes underlying emotional disorders.

Without belaboring the point, a construct with such a nomological network sits very far from an inclusive understanding of intelligence, let alone one that could begin to transcend 
Western-focused conceptions (Henrich, Heine, \& Norenzayan, 2010; Sternberg, 2000). It is urgent to work towards a more realistic conception of intelligence, which is the main objective of the present paper. Before we turn to it, however, it would be useful to speculate about what it is that IQ tests might really be measuring, since it should now be clear that it cannot be any cogent interpretation of intelligence.

IQ tests call upon one's capacity to act with maximal conformity and egocentricity at a particular point in time. This compound capacity is amoral and short-termist alike. Of course, it is also a capacity that, exercised consistently, increases the likelihood of a range of socially desirable outcomes, like civil obedience, educational achievement, and employment. While such statistical associations might perhaps be informative from a sociological perspective, they are of very limited value to the individual human being. Furthermore, IQ test scores have little relationship with key products of intellectuality, like creativity and genius (e.g., Guilford, 1967; Kim, 2005). They sometimes are at "actual retardation" levels in savant individuals, who, in fact, exhibit superlative intellectual abilities in a single or even multiple different areas, while being at normal or high levels in individuals who are "functionally retarded" (Treffert, 2014). High IQ is also patently inadequate as a predictor of socially significant eminence and success (Simonton, 2016).

There are innumerable ways to be intelligent and to act intelligently, the majority of which remain untapped by standardized IQ tests with their exclusive focus on algorithmic reasoning, linear thinking, memorization, and vocabulary size. The inevitable conclusion based on the foregoing discussion is that IQ is insufficient, often misleading, as a comprehensive index of intelligence. That said, it is not the main objective of this paper to join the chorus of criticism of IQ, but rather to introduce a new and complete conception of intelligence. 
RADIX INTELLIGENCE 8

\section{Radix Intelligence}

Radix Intelligence (RI) is the primal energy underpinning mind activity in its entirety. It is an elemental psychological quality within an idealist universe made out of ideas, rather than atoms. Radix Intelligence is intelligence and, ultimately, there is no intelligence other than Radix Intelligence. In the specific domain of human psychology, Radix Intelligence translates to a mental energy that is prior to all psychological traits. Etymologically, "radix" in Latin means "root" with a broader meaning of "primary source", while also carrying the useful connotation of

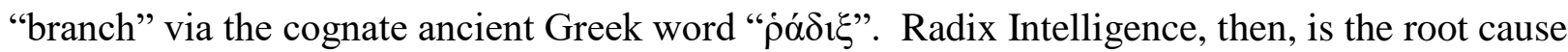
of all psychological traits as they manifest in ordinary experience. It guides the individual to adapt to the environment in a manner that maximizes, not conformity or survival chances, but psychological evolution.

While I am keen to avoid digressing into a discussion of construct labeling and its diverse effects (see Pace \& Brannick, 2010), it is relevant to record that several alternative labels are possible for this construct, the most appropriate of which is perhaps "Radix Energy". Still, the term 'energy' might imply closer links than is warranted with the psychodynamic theories of, mainly, Freud and Jung with their overlapping conceptions of psychic energy (see Petrides, 2019 for a brief discussion of these theories with reference to Psychobionomy). A foremost difference between Radix Intelligence and psychodynamic theories is that, with the notable exception of Wilhelm Reich's, they view energy as an exclusively mental concept to be distinguished in essence from its physical counterpart. By contrast, Radix Intelligence spans and permeates the physical and psychical. 'Intelligence,' furthermore, its connotations encompassing strategic and dynamic activity alike, is more apposite a term for the present purposes than 'energy,' which implies unstructured potentiality. Lastly, in relation to debates within differential psychology, 
usage in the label of the current conceptualization of the term 'intelligence' contributes to the broader endeavor to release it from the constrictive confines of IQ models.

Radix Intelligence unifies all desirable traits of the human mind and, by straightforward extension, all undesirable traits, casting them as expressions of low Radix Intelligence ${ }^{1}$. It is more general than Webb's $w$ (now subsumed under the General Factor of Personality; Musek, 2007; van der Linden et al., 2017), and its negative pole, the General Factor of Psychopathology (p; Caspi et al., 2014). It is also obviously more general than Spearman's $g$, which is fully reliant on a narrow type of psychometric methodology (maximal performance measurement) with which it has become virtually synonymous (Cronbach, 1960; Hostee, 2001) $)^{2}$ Indeed, the $g$ definition is so restricted in relation to Radix Intelligence that the entire theoretical structure of the former (Carroll, 1993) forms but a single solitary facet of the latter.

It should be clear that such a construct as I am describing here cannot be depicted graphically. Nevertheless, Figure 1 is an attempt to illustrate some general principles by means of a representational structure that allows for a psychometric approach to the subject. Three caveats should be made in relation to this figure. First, it is incomplete and incompletable. There is no rational basis for deciding what the main facets of Radix Intelligence are. This is because, as previously noted, Radix Intelligence permeates existence in its entirety, so it cannot, ultimately, be carved up into dimensions and facets. In this article, I am focusing exclusively on its structure and influence within the human psychological domain, although Radix Intelligence operates in all other life domains, including in animals, as well as in the physical world. That is

\footnotetext{
${ }^{1}$ As has been explained, Radix Intelligence is invariant and, strictly speaking, there is no question of it being high, average, or low. Nevertheless, the self-construct interferes with its constant projection, thus creating the perception of fragmentation into different areas (traits) as well as of variation and positioning on these traits (see Figures 1 and $3)$.

${ }^{2}$ Despite this severe mono-method bias, there is concrete evidence that the general factors of intelligence and personality are positively inter-correlated (Dunkel, 2013; Schermer \& Vernon, 2010).
} 
to say, the intelligence that is amply evident in the laws and functioning of the cosmos, is Radix Intelligence. It should, therefore, come as no surprise that such a construct cannot be fully illustrated.

Even within the borders of the domain of human psychology, it is impossible definitively to determine the facets of Radix Intelligence, something that has not even been achieved for conceptions that are far more limited and, basically, mere facets of it, like, for example, the " $\mathrm{g}$ factor" (Carroll, 1993) or the "Multiple intelligences" (Gardner, 2011). Furthermore, there is no factual process that can differentiate between potential facets and outcomes of Radix Intelligence. For instance, "successful intelligence" (Sternberg, 1999) could be seen as both a facet and an outcome of Radix Intelligence. Regardless, Figure 1 does cover the bulk of the Radix Intelligence domain in human psychology.

The second caveat about Figure 1, with definite consequences for the first, is that the structure it depicts is fluid and dynamic. Psychologically, none of these traits exists independently of the others. The notion that cognition is separate from, say, emotion, and emotion is separate from creativity is unsound. At any particular point in time, the intelligent response to a situation may be some emotional reaction (e.g., joy) or some creative act or intuitive perception, thus practically equating Radix Intelligence with one of its putative facets and conflating the entire figure to a single box. The hierarchical structure in Figure 1 is hypothetical and heuristic because the expression of Radix Intelligence does not unfold along independently preexisting trajectories or conjectural models. It is only the self-construct, cemented through repetitive patterns of cognition and behavior, and explored numerically in large multivariate samples (as opposed to psychologically in individual persons), that obstructs 
the ever-renewing flow of Radix Intelligence and creates the appearance of deeply rooted, clearly delineated and differentiable traits.

Stemming from the first two caveats is the third, viz., that the structure in Figure 1 is not necessarily recoverable in factor analyses and certainly not in the clinical neatness depicted in the figure. The objective of mentioning this caveat is to discourage mechanical factorizations and their attendant technical disputes that are so trivial psychologically. Radix Intelligence is the universal energy behind all aspects of existence with numerous theoretical and practical implications for human psychology. It should not be treated as yet another construct designed to be dissected, overanalyzed, and turned into a proliferating literature, but as a powerful explanatory framework within which to synthesize the glut of data dominating modern approaches to psychology with near-zero epistemological value and scant regard for interpretability from the perspective of the individual human being (Young, 2018).

Radix Intelligence differs radically from extant propositions within the wider field of intelligence. For one thing, it is not personal. Quite unlike any other conceptualization, from Spearman's (1904) g, to Guilford's (1967) structure of intellect model, Sternberg's (1999) successful intelligence, and Gardner's multiple intelligences (2011), Radix Intelligence permeates existence as a whole. The same intelligence that is evident everywhere in nature, also operates in animals and humans as part of nature. Individuals, assuming their existence (Parfit, 1984; Petrides, 2019), plug into this collective intelligence. Radix Intelligence is not a personal possession of the individual, a hypothesis that typically elicits the further speculation that it must be located somewhere in the individual's brain. Radix Intelligence is non-local, the brain being its mediator, rather than its generator. In fact, following Psychobionomy (Petrides, 2019), the brain is seen as a consequence of Radix Intelligence instead of its abode. 
Within the system of Psychobionomy (Petrides, 2019), Radix Intelligence is an integral aspect of the Generic Ground of Pure Knowing (Knowing). The four stages of human experience emanating from Knowing do so under lawful intelligence. That intelligence, which permeates the four stages of the Psychobionomic system, permeates, first and foremost, the human mind, including all of the attributes shown in Figure 1. The individual, like everything else in the phenomenal world, can never be severed from Radix Intelligence because he or she is a miniscule emanation of it. Indeed, from the higher perspective of Knowing, of which Radix Intelligence is but a feature, all there ever is Knowing, without individuals or other objects.

From the lower perspective of the individual, however, there exist separate human beings each of whom "possesses" (or, psychometrically speaking, "has a standing on") some degree of

the traits in Figure 1. It then behooves the psychometrician to measure those traits and, ideally, to build detailed theoretical accounts of them. The difficulty is that because psychometrics operates in stages 3 (Perceiving) and 4 (Acting) of the Psychobionomic system, it has no possibility of uncovering the complete true structure of the traits, whose origins lie mainly in the preceding Psychobionomic stages of Thinking and Feeling (Petrides, 2019). Consequently, it gets caught into a process of fragmentation, deficient or incomplete measurement, and, on occasion, profoundly questionable conclusions (as witnessed, for example, in the literature on IQ and race; Heinz, Müller, Krach, Cabanis, \& Kluge, 2014).

\subsection{Measurement of Radix Intelligence}

Once it manifests, Radix Intelligence is an absolute, not a relative phenomenon. For anything to be measurable, it ought to be finite; Radix Intelligence is infinite, therefore unmeasurable. It follows, as a corollary, that Radix Intelligence cannot be fruitfully investigated using the limited empirical methodologies to which limited conceptions of intelligence are 
subjected with varying degrees of cogency. The construct is offered with a view to informing the interpretation of the huge amounts of data that are already available, albeit compartmentalized and underutilized. Nevertheless, it is of some value to enquire whether we can approximate the extent to which it is mediated through (or reflected in) the activities of the human mind. Of course, the number of these activities is vast and their nature highly complex, such that significant simplification is required right at the outset of the process. Figure 1 presents the outcome of this simplification, depicting six distinct, yet interrelated, areas in which Radix Intelligence is reflected.

\subsubsection{Self-perceptions}

Having recommended an indefinite sampling domain for Radix Intelligence, in the form of the open structure in Figure 1, a suitable measurement strategy is required to simulate operationalization. Such a strategy should not only be able to provide reliable measurement for each of the areas in Figure 1, but also to move into the deeper segments of the mind, where Radix Intelligence tends to be less obstructed by overlaying objects. This is clearly depicted in the illustrating diagram of Psychobionomy (see Figure 1 in Petrides, 2019), where we observe that Knowing (of which Radix Intelligence is a property) is progressively transmuted into Thinking, Feeling, Perceiving, and finally, Acting. Measurement of such depth cannot be achieved in the outer periphery of the mind, but requires and demands the engagement of the individual's self-construct in the generation of numerical scores.

Self-reports have been variously used in the assessment of all areas in Figure 1 and this is indeed a methodology that forces the respondent to engage their self-construct in the formulation of their responses. The resultant responses reflect, to various degrees depending on the broader context, the respondent's fundamental perceptions of themselves and of the world. 
The process is illustrated in Figure 2 (panel A) where it is also juxtaposed against the standard IQ measurement process, involving the ability to "solve riddles per minute" (Heinz et al., 2014) and operating at a surface level of the mind, far away from the person's self-construct (panel B). The engagement of the self-construct via the expression of self-perceptions functions as a force of cohesion yielding the positive intercorrelations observed in self-report studies across the very diverse areas in Figure 1. This property of self-construct engagement is absent from IQ measurement, hence its resultant scores represent psychologically disjointed pieces of information that reveal very little that is dependable about the individual's inner being. What is more, even this information is ultimately opaque because it is unclear what it really reflects. In the words of the eminent intelligence researcher, Nathan Brody: "We know how to measure something called intelligence, but we do not know what has been measured" (Brody, 2000, p. $30)$.

The foregoing, including the differential measurement processes juxtaposed in Figure 2, account for the low observed correlations between IQ and many of the other traits and their facets depicted in Figure 1. The reason why IQ is not substantially correlated with those other traits or with life criteria like happiness and compassion, is because it is a relatively poor indicator of Radix Intelligence. IQ tests exhaust their assessment within the Psychobionomic stage of Acting, which lies on the outmost periphery of human experience. In other words, standard IQ testing makes no demand on the individual to engage with their self-construct out of which their whole personality essentially springs (see Figures 1 and 3 in Petrides, 2019). Inevitably, this means that IQ test scores will not be strongly associated with scores from measurement approaches that do engage the self-construct, even though significant intercorrelations are occasionally observed (e.g., Dunkel, 2013; Frederickson, Petrides, \& 
Simmonds, 2012) as we might expect for facets of the same overarching construct (Radix Intelligence).

When an attempt is made to filter the intelligence assessment through the individual's self-construct, as in self-report measures of intelligence, substantial and reliable correlations do arise with IQ test scores (Paulhus, Lysy, \& Yik, 1998) as well as with most other areas in Figure 1. It has also been found that self-estimates of various aspects of intelligence intercorrelate very strongly and cross-culturally (e.g., von Stumm, Chamorro-Premuzic, \& Furnham, 2009), although the source of these correlations has never been explicitly identified. Aspects of the present article illuminate this whole literature by identifying not only the source, but the very mechanism that produces these correlations, which is illustrated in Figure 3. It can be seen in that figure that the constant stream of Radix Intelligence is, in a sense, refracted by the individual's self-construct into the major trait dimensions illustrated in Figure 1. The attribute being refracted is coherently unitary, which guarantees that the products of the process, when suitably measured, will be empirically correlated. This is precisely what we observe in literally scores of research studies measuring these and related variables through self-report.

Self-report assessment can be performed with various degrees of consciousness (or indeed unconsciousness); additionally, variations in the solidity of an individual's self-construct will also complicate the interpretation of self-reports. In self-report operationalizations, measurement variance (which is typically, but not inevitably, classified as measurement error) is introduced by the variation in the degree of insight respondents have into their self-construct, a validity threat that has long been acknowledged (e.g., Wilson, Hull, \& Johnson, 1981) as well as by the solidity (robustness) of the self-construct itsel $\mathrm{f}$, a hitherto unacknowledged threat. The more robust (solid or opaque) a self-construct is, the greater its interference with the natural flow 
of Radix Intelligence and the harder it becomes to reach those of its facets that lie outside conscious awareness.

It is the presence of a personal self-construct that creates the concept of a trait as a consistent characteristic distinguishing one person from another. Radix Intelligence is the constant primal creative power projecting onto all domains of life as evolutionary cognitive, emotional, social, creative, etc. energy (see Figure 1). In the absence of a self-construct, it would be impossible to differentiate between all these manifestations of Radix Intelligence. Selfconstruct, which is the result of self-centeredness, is centrally involved in the fragmentation of the mind into multiple domains that, over time, become fixed as traits. These traits are generally consistent and habitual, rather than immutable, because self-constructs are non-invariant (i.e., they change over time and circumstances). Trait variability is the result, not of lability in the intensity of Radix Intelligence, but of changes in the underlying self-construct, which refracts Radix Intelligence into the multiplicity of areas shown in Figure 1. In parallel, because selfconstructs are fundamentally unstable, the resultant traits also have to be unstable.

In direct contrast to Radix Intelligence, which is invariant and always adaptive with respect to the individual's struggle for psychological evolution, in the phenomenal world of egoic action (i.e., the fourth Psychobionomic stage of Acting) we are left with traits whose stability and adaptative value both vary. Even assuming that adaptation is to be defined in the banal and culture-dependent terms of commonplace achievement (at school, work, etc.), conventional relationships, and longer lifespans, as it typically is in the peer-reviewed literature, the adaptive (or maladaptive) value of traits will vary as a function of context. This is widely discussed in relation to personality traits (e.g., Boyce, Wood, \& Brown, 2010; Friedman, 2019; Petrides, Vernon, Schermer, \& Veselka, 2011; Pettersson et al., 2014), but less so in relation to 
IQ, which is usually portrayed as some kind of adaptational panacea (e.g., Gottfredson, 1997; although see Charlton, 2009; Gignac \& Starbuck, 2019; Karpinski et al., 2018).

\subsubsection{Are high Radix Intelligence scores always adaptive?}

Radix Intelligence, it is worth reiterating, cannot be measured because it is not finite. Theoretically, it is an always-desirable quality and so, if its intensity did vary, more would always be better. However, the intensity of Radix Intelligence is invariant, which is why it may not be conceptualized as a trait in the traditional sense of a distinguishing characteristic. What does vary intensely is the solidity of the self-construct that refracts Radix Intelligence into traits as per the mechanism illustrated in Figure 3. It is, therefore, the separative presence of the illusory self-construct (see Petrides, 2019) that establishes the traits via its action as a labile refractor of the constant flow of Radix Intelligence.

Furthermore, Radix Intelligence is not aligned with the (commonly presumed) IQ advantage of routine adaptation to society (which, it should be remembered, is psychologically ailing; Vigo, Thornicroft, \& Atun, 2016). Rather, it is geared towards the psychological evolution of the individual with the ultimate aim of leading them to the transcendence of their self-construct. This is equivalent to the transcendence of personhood and of the separative perspective that lies at the very heart of human dysfunctionality. In the language of Psychobionomy (Petrides, 2019), it translates to psychological stabilization in (or identification with) the Generic Ground of Pure Knowing.

When it comes to the question of the adaptative value or desirability of particular traits, it should be understood that, unlike Radix Intelligence, no trait, irrespective of how desirable it might be in theory, will be universally advantageous in life. The reasons for this cannot be expounded in detail herein, except to mention that as distinguishing characteristics between 
individuals, all traits are contaminated by the limiting properties of the self-construct. Moreover, all evaluative judgements of what is desirable in the real world are temporally and geographically conditioned. Adaptive value is a conditional concept that can only be examined from the right side (output end) of Figure 3, not the left side (input end). The right side presents the gamut of traits that are the product of the refraction of Radix Intelligence by the individual's self-construct. Provided the assessment methodology passes through the individual's selfconstruct (Figure 2, panel A), its results will be informative in relation both to that self-construct itself and to the relative adaptive value of the traits to which it gives rise.

If the assessment methodology does not pass through the self-construct (Figure 2, panel B), as in the case of IQ testing, the results are bound to be unenlightening with respect to it. They may well convey information about other people's perceptions of the target individual's traits or their perceptions of whether the individual can successfully fulfil certain externally imposed expectations; however, they will be irrelevant for the individual's self-construct, which is what ultimately determines his or her overt behavior, unless he or she chooses to appropriate and internalize others' perceptions and evaluations of himself or herself. An individual who is able to withstand the impact of negative external evaluations can achieve astonishing and multifaceted eminence even with a scientifically measured IQ in the $16^{\text {th }}$ percentile (Harrison, 2001).

One drawback of eliciting trait responses via the individual's self-construct is that these cannot reveal the degree to which Radix Intelligence is allowed to operate in the individual. Put differently, these responses are uninformative with respect to the solidity of the self-construct. This is because traits are products of refraction, a type of distortion whose effects cannot be reverse-estimated (postdicted) using psychometric procedures. Perhaps this can be best 
explained with reference to the assessment of trait emotional intelligence (trait EI; Petrides, 2009), one of the major areas of expression of Radix Intelligence (see Figure 1).

There are three different pathways in which someone can achieve a high trait EI score. First, through dissimulation; while this is reliably picked up by sound applications of the TEIQue, flawed applications (e.g., slapdash, under-resourced, or by untrained individuals) pose a threat, especially in high-stakes assessments. In the second pathway, which is the commonest, a high trait EI score is achieved by means of an overblown self-construct. Here, the individual has a solid and inflated self-construct, largely derived through favorable comparisons against others. In the third pathway, which is comparatively rare, the individual has managed significantly to diminish, or altogether transcend, their self-construct, such that the influence of Radix Intelligence is reflected pure in their personality, which can result in extremely high trait EI scores. Because these three pathways (especially the second and third) cannot be psychometrically disentangled, it is impossible to estimate the robustness of the underlying selfconstruct (and, by inference, the strength of Radix Intelligence emanation) exclusively from psychometric profiles.

\subsection{Implications of Radix Intelligence}

The conception of Radix Intelligence is not simply original, but theoretically and practically consequential. Below, I sketch out some of its more significant implications and possibilities.

\subsubsection{Theoretical implications}

Radix Intelligence sparks crucial developments and opportunities for integration across the whole field of differential psychology. No longer are we encumbered by the fissures and divisions creating conceptual disintegration into a myriad of partly overlapping constructs 
(Shaffer, DeGeest, \& Li, 2016). Nor do we get mesmerized by the indiscriminate dataclysm that is inflating existing literatures and generating new ones without any hope of convergence. Appreciation of Radix Intelligence and of the manner in which it is refracted into the multitude of traits that are traditionally seen as, more or less, autonomous allows us to begin recognizing and, in due course, experiencing the essential unity underpinning the spectrum of activity of the human mind.

The refraction caused by the presence of the self-construct creates an array of major traits (see Figure 3). Aided and abetted by the application of inadequate or downright invalid psychometric methodologies, this array is subsequently shattered into innumerable little constructs. Insofar as these bitty constructs, by design or by chance, capture any aspect of the individual's self-construct, they will be intercorrelated. Actually, because Radix Intelligence is a universal - not personal - quality, one would expect all of its manifestations in the phenomenal world to be intercorrelated. It transpires that this is indeed the case, a fact long ago observed by statisticians (e.g. Berkson, 1938). In social science debates, it emerged under the most unfortunate label “crud factor” introduced by Meehl (1990; see also Lykken’s, 1968 “ambient correlational noise") who interpreted it as some kind of problem in need of resolution. The socalled "crud factor" is nothing other than the Radix Intelligence signal as detected by the crude measurement methodologies of the social sciences. That it has been misinterpreted as "crud" is puzzling because, even in the absence of the Radix Intelligence insight, it ought to have been noted, at the point of choosing a label for this phenomenon, that systematic covariation does not just pop up out of noise.

On a related note, measurements that have been filtered through the individual's selfconstruct (Figure 2, panel A), should be expected to show still greater intercorrelations. This 
turns out to be fully supported by the data as is evident in the general and higher-order factors emerging in self-report measurements of (supposedly orthogonal) personality dimensions, with a regularity that has prompted calls for orthogonality to be strategically engineered into datasets (Saucier, 2002). More generally, the correlations consistently arising between self-report measures have engendered the large literature on common method variance ${ }^{3}$ and its armamentarium of remedies (Campbell \& Fiske, 1959), the presumption being that the source of the overlapping variance is a methodological artifact that ought to be controlled for at the stages of design or analysis. When it comes to self-perceptions, however, common method variance is pregnant with evidence about the underlying self-construct that needs to be processed and understood (above all, by the individual concerned) instead of statistically eliminated as if it were some kind of inconvenient confounder.

\subsubsection{Practical implications}

There are important practical advantages to be gained from the understanding of Radix Intelligence, especially as framed within the overarching system of Psychobionomy. A foremost example from the domain of psychometrics, concerns the possibility of low-, minimal-, or even zero-information personality assessment (LIPA, MIPA, or ZIPA) - 'information' here specifically referring to psychometric data. This process involves the ability to extract personality and behavioral information based on the recognition of the self-construct as a refractor of Radix Intelligence. By identifying the underlying beliefs that establish the

\footnotetext{
${ }^{3}$ Incidentally, common method variance is routinely ignored in IQ research despite its pervasive presence in key domains of validation like academic and job performance, where there is the ubiquitous occurence of cognitive ability tests predicting educational attainment tests and training performance tests ("tests predicting tests"; Goldstein, Zedeck, \& Goldstein, 2002). Self-perceptions, on the other hand, are generated and sustained by the selfconstruct, which renders it, not self-report methodology per se, the cause of their observed intercorrelations. If selfreport methodology were the true cause of these intercorrelations, then we would expect all variables measured by self-report to be significantly intercorrelated, which is far from the case for either self-report or other measurement methods (Spector, 2006).
} 
boundaries of the self-construct, which acts as a refractor of Radix Intelligence, it is possible to make inferences about someone's personality traits and potential behaviors. The assessment of these beliefs can be carried out even in the absence of conventional psychometric data as indirectly, partially, and unintentionally effected in personality profiling and behavioral prediction from digital footprints (Azucar, Marengo, \& Settanni, 2018; Schoen et al., 2013).

Radix Intelligence (and, more broadly, Psychobionomic) applications promise to complement data-driven prediction with theory-driven prediction, while simultaneously reducing, or altogether removing, traditional limitations in psychometrics. Some such are prohibitively long testing times, reliance on transparent items as well as impotence in detecting the right constructs for prediction across different contexts and in assessing dispositions that lie outside conscious awareness. The last one, especially, offers rich opportunities for the advancement of a whole new field, under the auspices of Psychobionomy, that may be termed “subconscious psychometrics".

The establishment of this new field will yield practical breakthroughs to address critical blind spots in psychological assessment practices. These currently include the partial or wholesale neglect of latent urges and fears, defense mechanisms, automatic behaviors, and generally any kind of influence on people's thoughts, feelings, perceptions, and actions that cannot be tapped through standard assessments of the conscious elements of personality, which are additionally passed through impression management filters. Subconscious effects (and also dark trait tendencies that are habitually concealed in psychometric assessments) have a decisive impact on all aspects of behavior over the long-term and thence on life trajectories as a whole. While these are well recognized in clinical and counselling settings, they remain overlooked in 
most others and a compelling area of challenge for contemporary psychometrics and related fields (Pratt \& Crosina, 2016).

Understood in the fullness of its potential, Radix Intelligence has radical consequences for what can be termed the psychology of becoming. This is a psychology centered on a neverending effort to improve the self-construct using a bewildering range of techniques and interventions that has swollen to such an extent that their mere taxonomization presents significant challenges (Michie et al., 2013). The problem is that all behavioral change efforts are caught in a self-sustaining loop, whereby limitations are assimilated into the self-construct ${ }^{4}$ through the very effort it expends to overcome them. One of the most pernicious side-effects of goal achievement using behavioral change mechanisms is the self-perpetuation of their operation through the erroneous impression that effort by the self-construct can remove limitations from the self-construct. No sooner than a particular limitation is overcome through some onerous technique or intervention, than the self-construct turns its attention to the next one, trapping the individual in an endless process of becoming.

Petrides (2019) describes an alternative process to behavioral change approaches, which, in their attempt to modify the self-construct, always end up enhancing its dividing presence and influence. The infolding process of involution through the four Psychobionomic stages seeks to direct the attention of the individual towards the core of their being, where they can discover the existence of their self-construct and its multifarious functions. Upon this discovery, the individual can endeavor either to operate directly on the self-construct, thus escaping the tremendous waste of effort in attempts to resolve challenges located near his or her psychological

\footnotetext{
${ }^{4}$ The self-construct is simultaneously the original limitation as well as the foundation for all limitations that are subsequently appended onto it.
} 
periphery (i.e., the standard modus operandi of behavioral change programs) or to transcend it, thus permanently liberating himself or herself from its limiting influence.

The psychology of becoming stands in stark contrast to the psychology of being, introduced and advocated in the system of Psychobionomy. This invites the individual to recognize and transcend the limiting mechanism of the self-construct beyond which lies the psychological simplicity and fulfilment of conscious being (i.e., the Generic Ground of Pure Knowing). Stabilization in Knowing, which entails establishing an inner identity as Knowing, rather than as the self-construct, entirely dispenses with the need for becoming. This transition into a psychology of being is to be realized by means of the progressive deconditioning of the mind from its entrenched experience of psychological severalty and effortfulness that are the key products of false identification with the illusory and limiting self-construct.

\section{Conclusion}

Following the principles of Psychobionomy (Petrides, 2019), this paper presents a new, and final - in the sense of all-encompassing - conception of intelligence. Radix Intelligence integrates not only previous conceptions, including the $g$ factor, but also the domain traditionally defined as personality. It is the primal energy behind all mind activity, which, in the realm of human psychology, is strikingly expressed in the gamut of traits and abilities researched under the rubric of differential psychology (Figure 1).

Radix Intelligence offers a unique possibility for the theoretical integration of all major individual differences dimensions that are currently scattered and on a steadfast course of fragmentation. Through its causal mechanism of self-construct refraction (Figure 3), the formulation holds considerable further promise for opening up avenues of process-focused personality explanations that move beyond the "verbal magic" offerings of models in the style of 
the Big Five. In turn, this can contribute towards remedying the inadequacy of most personality traits as coherent explanatory constructs (Boag, 2011).

From an applied and practical perspective, Radix Intelligence, within the broader context of Psychobionomy, presents opportunities for psychological emancipation through the transcendence of the self-construct with all its distorting and limiting effects. This does not involve psychometric and statistical algorithms applied by experts to large groups of anonymous and disempowered individuals, but a revolution of identity and of values effected by the person himself or herself and affecting his or her existence, not in piecemeal manner as in behavioral change programs, but in its totality. A separate domain of applications can focus on longedcraved psychometric breakthroughs in the development of novel and efficient methodologies for the holistic assessment of personality, including its subconscious components.

The inherent resistance of the self-construct to renouncing those beliefs and activities that sustain its illusory existence and its domination of the individual's personality is the sole obstacle to experiencing the transformative effects of Radix Intelligence that are so sorely needed in everyday life. It was the main aim of this paper to introduce the nature and properties of this genuinely general, complete, and invariant conception of intelligence. I hereby commend it, collectively, to the scientific and educational communities for theoretical, research, and practical purposes, and, individually, to each person wishing to work towards establishing an inner psychology of being. 


\section{References}

Azucar, D., Marengo, D., \& Settanni, M. (2018). Predicting the Big 5 personality traits from digital footprints on social media: A meta-analysis. Personality and Individual Differences, 124, 150-159.

Bandura, A. (1999). Social cognitive theory of personality. In L. A. Pervin \& O. P. John (Eds.), Handbook of personality: Theory and research. New York, NY, US: Guilford Press.

Berkson, J. (1938). Some difficulties of interpretation encountered in the application of the chisquare test. Journal of the American Statistical Association, 33, 526-536.

Boag, S. (2011). Explanation in personality psychology: "Verbal magic" and the fivefactor model. Philosophical Psychology, 24, 223-243.

Boring, E. G. (1923). Intelligence as the tests test it. New Republic, 36, 35-37.

Boyce, C. J., Wood, A. M., \& Brown, G. D. (2010). The dark side of conscientiousness: Conscientious people experience greater drops in life satisfaction following unemployment. Journal of Research in Personality, 44, 535-539.

Brody, N. (2000). History of theories and measurements of intelligence. In R. J. Sternberg (Ed.), Handbook of intelligence (pp. 16-33). NY: CUP.

Campbell, D. T. \& Fiske, D. W. (1959). Convergent and discriminant validation by the multitrait-multimethod matrix. Psychological Bulletin, 56, 81-105.

Carroll, J. B. (1993). Human cognitive abilities: A survey of factor-analytic studies. New York: CUP.

Caspi, A., Houts, R. M., Belsky, D. W., Goldman-Mellor, S. J., Harrington, H., Israel, S., ... \& Moffitt, T. E. (2014). The p factor: one general psychopathology factor in the structure of psychiatric disorders? Clinical Psychological Science, 2, 119-137. 
Cassidy, R. M., Yang, F., Kapczinski, F., \& Passos, I. C. (2017). Risk factors for suicidality in patients with schizophrenia: a systematic review, meta-analysis, and meta-regression of 96 studies. Schizophrenia Bulletin, 44, 787-797.

Charlton, B. G. (2009). Clever sillies: Why high IQ people tend to be deficient in common sense. Medical Hypotheses, 73, 867-70.

Cronbach, L. J. (1960). Essentials of psychological testing (2nd ed.). New York: Harper \& Row.

Dunkel, C. S. (2013). The general factor of personality and general intelligence: Evidence for substantial association. Intelligence, 41, 423-427.

Fisher, A. J., Medaglia, J. D., \& Jeronimus, B. F. (2018). Lack of group-to-individual generalizability is a threat to human subjects research. Proceedings of the National Academy of Sciences, 115, E6106-E6115.

Frederickson, N., Petrides, K. V., \& Simmonds, E. (2012). Trait emotional intelligence as a predictor of socioemotional outcomes in early adolescence. Personality and Individual Differences, 52, 317-322.

Friedman, H. S. (2019). Neuroticism and health as individuals age. Personality Disorders: Theory, Research, and Treatment, 10, 25-32.

Gardner, H. (2011). Frames of mind: The theory of multiple intelligences. New York: Basic Books.

Gignac, G. E., \& Starbuck, C. L. (2019). Exceptional intelligence and easygoingness may hurt your prospects: Threshold effects for rated mate characteristics. British Journal of Psychology, 110, 151-172.

Goldstein, H. W., Zedeck, S., \& Goldstein, I. L. (2002). g: Is this your final answer? Human Performance, 15, 123-142. 
Goriounova, N. A. \& Mansvelder, H. D. (2019). Genes, cells and brain areas of intelligence. Frontiers in Human Neuroscience, 13, 44. doi: 10.3389/fnhum.2019.00044

Gottfredson, L. S. (1997). Why g matters: The complexity of everyday life. Intelligence, 24, 79132.

Gould, S. J. (1996). The mismeasure of man (2nd ed.). N.Y.: Norton.

Guilford, J. P. (1967). The nature of human intelligence. NY: McGraw-Hill.

Harrison, B. T. (2001). The Muhammad Ali draft case and public debate on the Vietnam War. Peace Research, 33, 69-86.

Heinz, A., Müller, D. J., Krach, S., Cabanis, M., \& Kluge, U. P. (2014). The uncanny return of the race concept. Frontiers in Human Neuroscience, 8: 836.

Henrich, J., Heine, S. J., \& Norenzayan, A. (2010). The weirdest people in the world? Behavioral and Brain Sciences, 33, 61-83.

Hofstee, W. K. B. (2001). Intelligence and personality: Do they mix? In J. M. Collis, \& S. Messick (Eds.), Intelligence and personality. Bridging the gap in theory and measurement. (pp. 43 - 60). Hillsdale, NJ: Lawrence Erlbaum Associates.

Howe, M. J. A. (1988). Intelligence as an explanation. British Journal of Psychology, 79, 349360.

Howe, M. J. A. (1997). IQ in question: The truth about intelligence. London: Sage.

James, W. (1890). The principles of psychology. New York: Holt.

Jensen, A. (1998). The g factor. Westport, CT: Praeger.

Joober, R. (2016). Deconstructing the mental health crisis in only 2 pieces. Journal of Psychiatry \& Neuroscience, 41, 222-224.

Jung, C. G. (1939). The integration of personality. New York: Farrar and Rinehart. 
Jung, R. E., \& Haier, R. J. (2007). The Parieto-Frontal Integration Theory (P-FIT) of intelligence: converging neuroimaging evidence. Behavioral and Brain Sciences, 30, $135-154$.

Karpinski, R. I., Kolb, A. M. K., Tetreault, N. A., \& Borowski, T. B. (2018). High intelligence: A risk factor for psychological and physiological overexcitabilities. Intelligence, 66, 823.

Kim, K. H. (2005). Can only intelligent people be creative? A meta-analysis. Journal of Secondary Gifted Education, 16, 57-66.

Kovacs, K., \& Conway, A. R. (2019). A unified cognitive/differential approach to human intelligence: Implications for IQ testing. Journal of Applied Research in Memory and Cognition, 8, 255-272.

Kreitler, S. (2019). Towards a consensual model in personality psychology. Personality and Individual Differences, 147, 156-165.

Lamiell, J. T. (2003). Beyond individual and group differences: Human individuality, scientific psychology, and William Stern's critical personalism. Thousand Oaks, CA: Sage.

Lopez, C., Stahl, D., \& Tchanturia, K. (2010). Estimated intelligence quotient in anorexia nervosa: a systematic review and meta-analysis of the literature. Annals of General Psychiatry, 9, 40.

Lykken, D. T. (1968). Statistical significance in psychological research. Psychological Bulletin, 70, 151-159.

Magnusson, D. \& Torestad, B. (1993). A holistic view of personality: A model revisited. Annual Review of Psychology, 44, 427-452. 
Meehl, P. E. (1990). Why summaries of research on psychological theories are often uninterpretable. Psychological Reports, 66, 195-244.

Michie, S., Richardson, M., Johnston, M., ... \& Wood, C. E. (2013). The behavior change technique taxonomy (v1) of 93 hierarchically clustered techniques: building an international consensus for the reporting of behavior change interventions. Annals of Behavioral Medicine, 46, 81-95.

Musek, J. (2007). A general factor of personality: Evidence for the Big One in the five-factor model. Journal of Research in Personality, 41, 1213-1233.

Pace, V. L. \& Brannick, M. T. (2010). How similar are personality scales of the "same" construct? A meta-analytic investigation. Personality and Individual Differences, 49, 669-676.

Parfit, D. (1984). Reasons and persons. Oxford: OUP.

Paulhus, D. L., Lysy, D. C., \& Yik, M. S. (1998). Self-report measures of intelligence: Are they useful as proxy IQ tests? Journal of Personality, 66, 525-554.

Penney, A. M., Miedema, V. C., \& Mazmanian, D. (2015). Intelligence and emotional disorders: Is the worrying and ruminating mind a more intelligent mind? Personality and Individual Differences, 74, 90-93.

Petrides, K. V. (2009). Psychometric properties of the Trait Emotional Intelligence Questionnaire. In C. Stough, D. H. Saklofske, and J. D. Parker, Advances in the assessment of emotional intelligence. New York: Springer. DOI: 10.1007/978-0-38788370-0_5

Petrides, K. V. (2019). A conceptual application of Psychobionomy to the field of personality and individual differences. Personality and Individual Differences, 147, 135-143. 
Petrides, K. V., Vernon, P. A., Schermer, J. A. \& Veselka, L. (2011). Trait emotional intelligence and the Dark Triad traits of personality. Twin Research and Human Genetics, 14, 35-41.

Pettersson, E., Mendle, J., Turkheimer, E., Horn, E. E., Ford, D. C., Simms, L. J., \& Clark, L. A. (2014). Do maladaptive behaviors exist at one or both ends of personality traits? Psychological Assessment, 26, 433.

Pratt, M. G. \& Crosina, E. (2016). The nonconscious at work. Annual Review of Organizational Psychology and Organizational Behavior, 3, 321-347.

Revelle, W. (2012). Integrating personality, cognition and emotion: Putting the dots together? In M. W. Eysenck, M. Fajkowska, \& T. Maruszewski (Eds.), Personality, cognition and emotion. Clinton Corners, NY: Eliot Werner.

Richardson, K. (2002). What IQ tests test. Theory \& Psychology, 12, 283-314.

Savage, J. E., Jansen, P. R., Stringer, S., Watanabe, K., Bryois, J., De Leeuw, C. A., ... \& Grasby, K. L. (2018). Genome-wide association meta-analysis in 269,867 individuals identifies new genetic and functional links to intelligence. Nature Genetics, 50, 912-919.

Saucier, G. (2002). Orthogonal markers for orthogonal factors: The case of the Big Five. Journal of Research in Personality, 36, 1-31.

Schermer, J. A. \& Vernon, P. A. (2010). The correlation between general intelligence (g), a general factor of personality (GFP), and social desirability. Personality and Individual Differences, 48, 187-189.

Schoen, H., Gayo-Avello, D., Takis Metaxas, P., Mustafaraj, E., Strohmaier, M., \& Gloor, P. (2013). The power of prediction with social media. Internet Research, 23, 528-543. 
Shaffer, J. A., DeGeest, D., \& Li, A. (2016). Tackling the problem of construct proliferation: A guide to assessing the discriminant validity of conceptually related constructs. Organizational Research Methods, 19, 80-110.

Simonton, D. K. (2016). Reverse engineering genius: historiometric studies of superlative talent. Annals of the New York Academy of Sciences, 1377, 3-9.

Spearman, C. (1904). “General Intelligence,” objectively determined and measured. American Journal of Psychology, 15, 201-292.

Spearman, C. (1927). The abilities of man. London: MacMillan.

Spector, P. E. (2006). Method variance in organizational research: truth or urban legend? Organizational Research Methods, 9, 221-232.

Sternberg, R. J. (1999). The theory of successful intelligence. Review of General Psychology, 3, 292-316.

Sternberg, R. J. (2000). The concept of intelligence. In R. J. Sternberg (Ed.), Handbook of intelligence (pp. 3-15). New York: CUP.

Thorndike, E. L. (1921). Intelligence and its measurement: A symposium-I. Journal of Educational Psychology, 12, 124.

Treffert, D. A. (2014). Savant syndrome: Realities, myths and misconceptions. Journal of Autism and Developmental Disorders, 44, 564-571.

Van der Linden, D., Pekaar, K., Bakker, A. B., Aitken Schermer, J., Vernon P. A., \& Petrides, K. V. (2017). Overlap between the general factor of personality and emotional intelligence: A meta-analysis. Psychological Bulletin, 143, 36-52.

Vigo, D., Thornicroft, G., \& Atun, R. (2016). Estimating the true global burden of mental illness. The Lancet Psychiatry, 3, 171-178. 
Von Stumm, S., Chamorro-Premuzic, T., \& Furnham, A. (2009). Decomposing self-estimates of intelligence: Structure and sex differences across 12 nations. British Journal of Psychology, 100, 429-442.

Webb, E. (1915). Character and intelligence: An attempt at an exact study of character. British Journal of Psychology Monograph Supplements, 1 (III), 1-99.

Wilson, T. D., Hull, J. G., \& Johnson, J. (1981). Awareness and self-perception: Verbal reports on internal states. Journal of Personality and Social Psychology, 40, 53-71.

Young, J. L. (2018). The long history of big data in psychology. The American Journal of Psychology, 131, 477-482. 
Figure 1. Representational Structure of Radix Intelligence in the Domain of the Personalized Human Mind

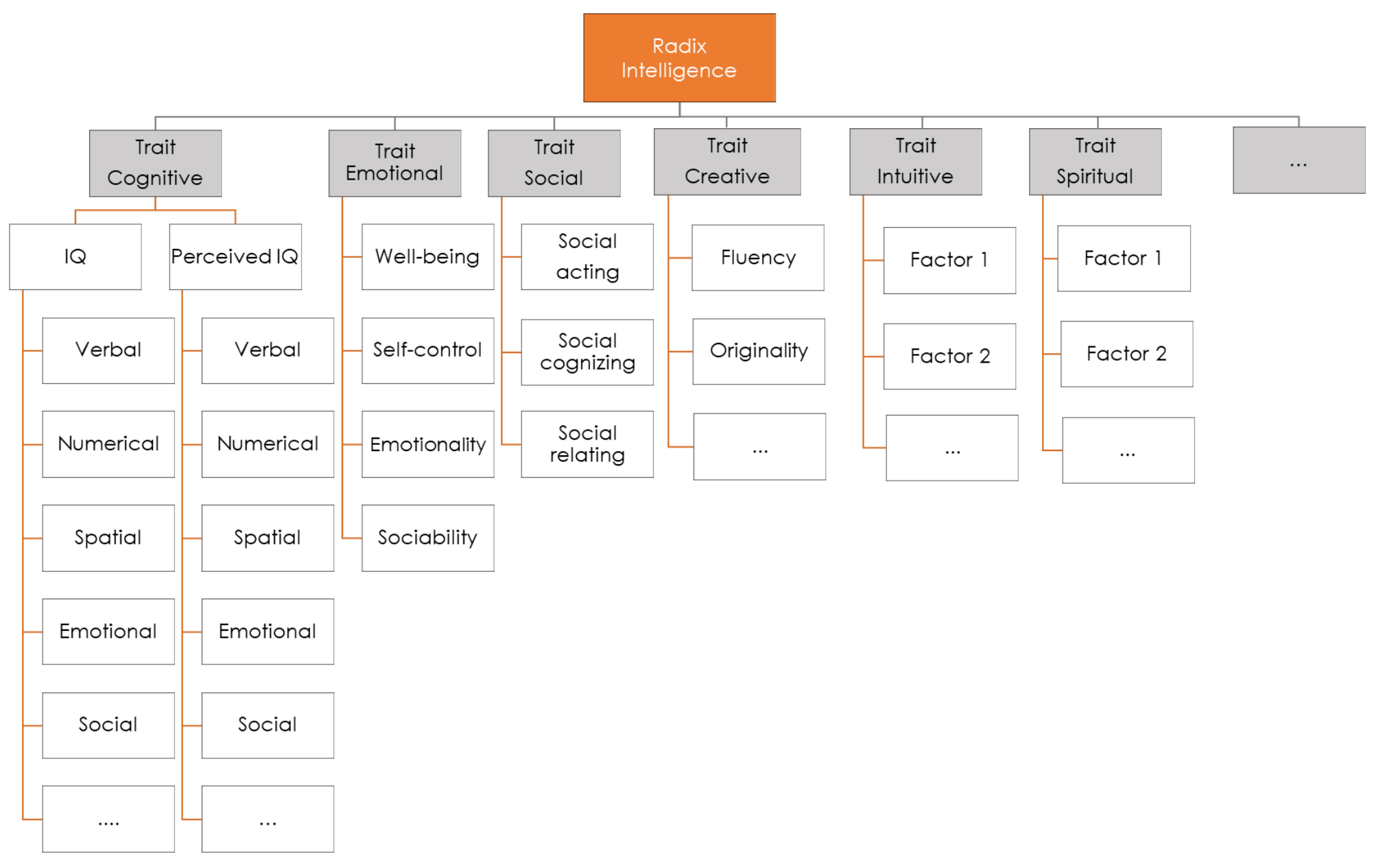


Figure 2. Psychological Assessment Passing through the Individual's Self-Construct (Panel A) versus Psychological Assessment Bypassing the Individual's Self-Construct (Panel B)

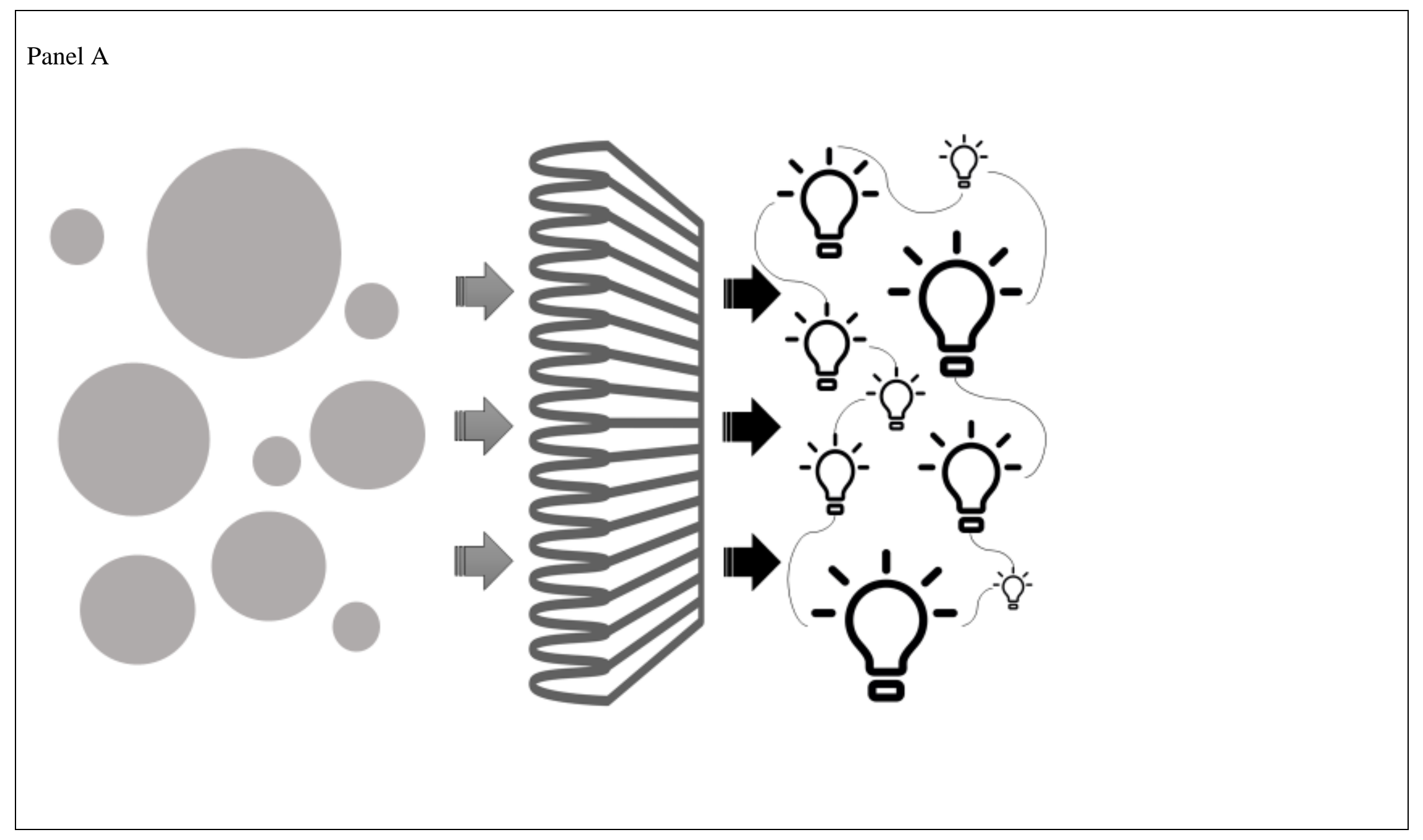




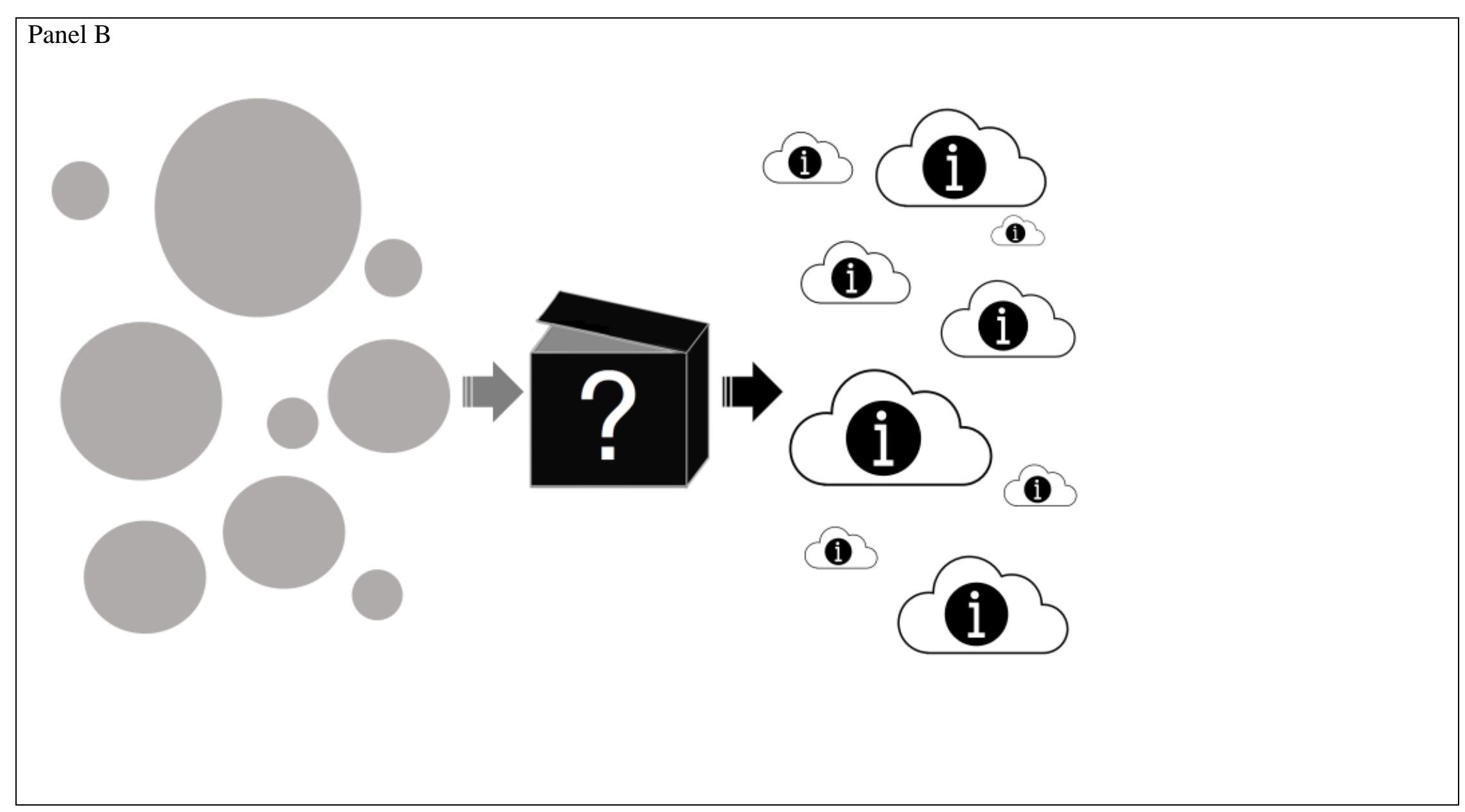




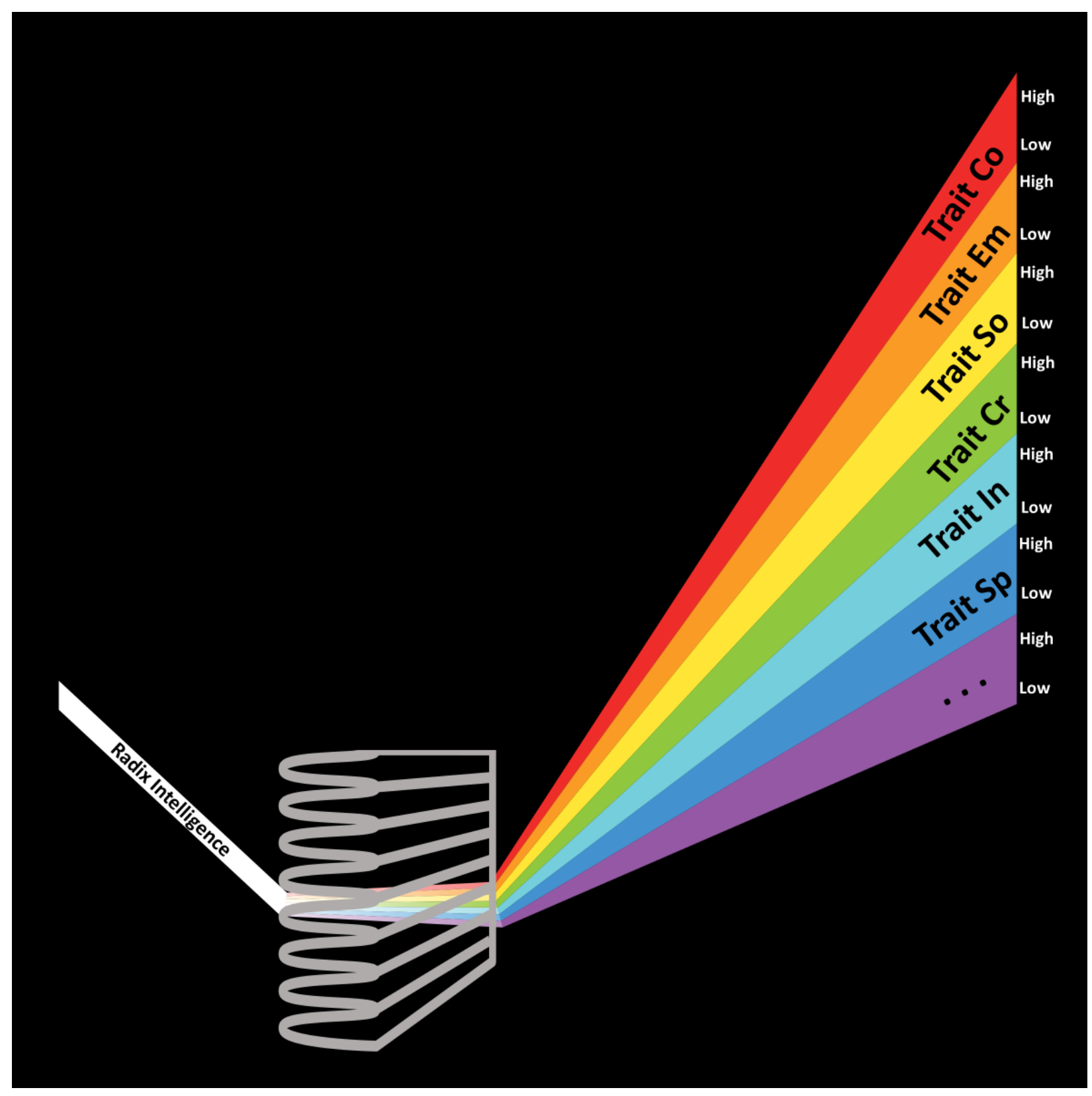




\section{Figure Captions}

Figure 1. The illustration is intended to convey general principles and, as such, entails a degree of fluidity in its application. Radix Intelligence is reflected in all major areas of human mind activity. Six such areas are depicted in the figure (trait cognitive, emotional, social, creative, intuitive, and spiritual intelligence), but many more exist. All these areas are traits, defined as consistent characteristics distinguishing one person from another and arising from the self-construct's refraction of the Radix Intelligence flow (see Figure 3). Each area comprises a number of factors, examples of which have been included in the figure, except in the cases of trait emotional and trait social intelligence, where the factor structures are settled. Special mention ought to be made to trait cognitive intelligence, which has been divided into IQ and perceived IQ. This methodological distinction cannot be drawn with confidence in areas of Radix Intelligence that are not readily amenable to maximum performance measurement (see, e.g., Cronbach, 1960 in relation to social intelligence). Within trait cognitive intelligence, perceived IQ is a better conduit of Radix Intelligence than test-based IQ because the former is filtered through the individual's self-construct and, consequently, yields insights that are more informative from a psychological perspective (see Figure 2). (C) K.V. Petrides, 2019 - London Psychometric Laboratory. All rights reserved. 
RADIX INTELLIGENCE 39

Figure 2. Assessment Passing through the Individual's Self-Construct (Panel A) versus Assessment Bypassing the Individual's Self-Construct (Panel B)

Circles on the left represent stimuli in test materials (IQ test items, questionnaire items, etc.). Psychometric assessment passing through the individual's self-construct (Panel A) yields insights about the self-construct in the form of self-perceptions. Through an exploration of the individual's self-perceptions, inferences can be made about the underlying self-construct, which has a causal influence on his or her life by affecting cognition, emotion, perception, and action. Furthermore, the insights revealed by this process, because they are reflective of the underlying self-construct, are interlinked across all major areas of human psychology, irrespective of how dissimilar and unrelated these may appear to be prima facie. One way in which the positive reintegration of personality (e.g., James, 1890; Jung, 1939; Magnusson \& Torestad, 1993) may be achieved, is through the integrative power of self-perceptions that extends into every area in which they are expressed. In contrast, psychometric assessment that bypasses the self-construct (Panel B) operates like a black box within the periphery of personality and, accordingly, yields information that is disjointed and shrouded in conceptual ambiguity. IQ scores show weak and erratic correlations with core aspects of personality despite the undeniable entwinement of intelligence and personality at the level of the individual organism. Thus, it is no wonder that the essence of IQ scores remains mysterious even after over a century of research (Brody, 2000; Richardson, 2002). (C K.V. Petrides, 2019 - London Psychometric Laboratory. All rights reserved. 
Figure 3. The Refraction of Radix Intelligence through the Individual's Self-Construct

Radix Intelligence is an aspect of the Generic Ground of Pure Knowing (Knowing) as described in the system of Psychobionomy (Petrides, 2019). The four stages of human experience that emerge out of Knowing (Thinking, Feeling, Perceiving and Acting) do so under the lawful influence of Radix Intelligence, which is an undifferentiated emanation of Knowing. In the absence of a self-construct, Radix Intelligence unfolds without boundaries and categorizations, continuously directing the totality of being to its optimal manifestation. However, the presence of a self-construct, emerging in the first stage of the Psychobionomic system (Thinking), refracts the unitary flow of Radix Intelligence into a manifold of major traits that can then be subdivided into various levels of granularity. Traits require the self-construct for their emergence, with the latter also governing their intensity (from relatively low to relatively high). Because self-constructs are in flux, the standing of individuals on the various traits is bound to show variability. With respect to the adaptive value of traits, unlike that of Radix Intelligence, it fluctuates across contexts, such that there are no traits that are reliably adaptive in all circumstances or whereon high scores are invariably desirable. Note: Trait Co $=$ Trait Cognitive Intelligence; Trait Em $=$ Trait Emotional Intelligence; Trait So $=$ Trait Social Intelligence; Trait $\mathrm{Cr}=$ Trait Creative Intelligence; Trait $\mathrm{In}=$ Trait Intuitive Intelligence; Trait Sp $=$ Trait Spiritual Intelligence. @ C K.V. Petrides, 2019 - London Psychometric Laboratory. All rights reserved. 